\title{
HIV Care Continuum and COVID-19 Outcomes Among People Living with HIV During the COVID-19 Pandemic, Chicago, IL
}

\author{
Jessica P. Ridgway ${ }^{1} \cdot$ Jessica Schmitt ${ }^{1} \cdot$ Eleanor Friedman ${ }^{1} \cdot$ Michelle Taylor $^{1} \cdot$ Samantha Devlin $^{1} \cdot$ Moira McNulty $^{1}$. \\ David Pitrak ${ }^{1}$
}

Published online: 7 May 2020

○) Springer Science+Business Media, LLC, part of Springer Nature 2020

The COVID-19 pandemic has the potential to lead to serious negative health outcomes for people living with HIV (PLWH). Besides the risk of COVID-19 disease itself, the indirect effects of the pandemic including shelter-in-place orders, record unemployment, and widespread social anxiety may interact synergistically to worsen health outcomes for PLWH $[1,2]$. In this report, we share the experience from University of Chicago Medicine regarding the early impact of the COVID-19 pandemic on PLWH in regards to retention in care, antiretroviral adherence, and COVID-19 testing and clinical outcomes.

\section{Retention in Care}

Retention in care is essential for the health of PLWH and for public health [3]. Patients who are retained in care are more likely to adhere to antiretroviral therapy and experience improved health outcomes, and are less likely to transmit HIV to others [4]. Missed medical appointments are independently associated with increased risk of AIDS-defining illnesses and death [5, 6]. Traditionally, retention in care has relied on the ability of PLWH to regularly attend in-person appointments with their HIV medical team, which is difficult during the COVID-19 pandemic.

To slow the spread of COVID-19, the Centers for Disease Control and Prevention (CDC) has strongly encouraged social distancing [7] and many U.S. state governments have imposed shelter-in-place or "lockdown" orders [8-10]. In response, the HRSA Ryan White Program has encouraged the use of telehealth to allow PLWH to stay home and not travel to clinics for HIV care visits [11], and many HIV

Jessica P. Ridgway

jridgway@medicine.bsd.uchicago.edu

1 Department of Medicine, University of Chicago, Chicago, IL, USA medical providers have moved to virtual medical visits utilizing telephone calls or online video conferencing services.

While virtual visits are helpful for some PLWH $[12,13]$, others may may not have reliable internet or mobile phone services to utilize these services [14]. Moreover, during the pandemic, many Infectious Diseases physicians and clinics are overwhelmed with COVID-19 patients, and lack the time or resources to respond to PLWH who may have additional needs or concerns during this time. Services and resources which in ordinary times are easily available may have been discontinued, with effects on reengagement in care and extension of prescriptions. Even if telephone or video conferencing visits are occurring, these visits may lack wrap around services provided by social workers and other HIV care team members.

Our medical center serves a predominantly African American population from economically disadvantaged areas on the south side of Chicago and is a major provider of HIV care in our community [15]. We began the process of converting in-person HIV care visits to telephone or video conferencing calls immediately after the state of Illinois imposed a "shelter in place" order on March 21, 2020 [8]. To best meet the needs of our patients, our clinic utilized a two-tiered approach of either delaying visits several months in the future, or arranging virtual visits. During the first 4 weeks of the order (March 21, 2020 through April 17, 2020), 98 PLWH were scheduled for HIV care visits in our clinic. Of these, $2.0 \%$ (2/98) attended in-person visits, $21.4 \%$ (21/98) attended virtual visits with a member of the HIV care team, and 30.6\% (30/98) rescheduled for a later date. Among the 45 PLWH who neither attended virtual visits nor were rescheduled, $60 \%(27 / 45)$ had a most recent HIV viral load (VL) < 20 copies/mL, 31.1\% (14/45) had VL between 20 and 200 copies $/ \mathrm{mL}$, and $8.9 \%$ (4/45) patients had VL $>200$ copies/mL. PLWH whose appointments were rescheduled or cancelled were more likely to have $\mathrm{VL}<20$ copies/mL than those who attended in-person or virtual 
visits $(89.3 \%,(67 / 75)$ vs. $39.1 \%,(9 / 23))$. This finding is encouraging, suggesting that providers prioritized visits with patients with detectable viral loads who were most in need of close follow-up. Ideally all patients would have the opportunity to connect with their HIV care providers, and we are in the process of reaching out to PLWH who neither attended visits nor rescheduled to arrange virtual visits.

\section{ART Access and Adherence}

Limited access and adherence to antiretroviral therapy are other possible hazards for PLWH during COVID-19. The pandemic has led to record-breaking unemployment claims and financial stress [16], including loss of health insurance, increased food insecurity, and fears of homelessness. Direct loss of income and prescription coverage may lead to PWLH not being able to afford or access medications. State AIDS Drugs Assistance Programs (ADAP) have recognized this barrier and modified the criteria for renewals and loosened the restrictions for submission [11]. Our clinic has increased mail delivery for prescriptions and extended refills while patients are not able to come in for laboratory testing. Our social work team has provided gift cards for grocery and supply stores in order to combat food insecurity—mailing or dropping off resources as needed.

Worsening of behavioral health disorders in the setting of the COVID-19 pandemic can also lead to decreased ART adherence. Many PLWH are disproportionately affected by mental health and substance use disorders [17-22], which may be exacerbated due to the uncertainty and anxiety caused by the pandemic [23]. We have mobilized our behavioral health program to provide more frequent, brief tele-therapy supports to provide continuity of mental healthcare. Although some patients prefer in-person contact and have temporarily discontinued services, we have seen in an increase in participation from patients who historically have not engaged in therapy services. Our programs have also created interactive video conference workshops and question and answer sessions for patients and community members who do not want to engage in individual therapy, but are in need additional support during this time.

\section{COVID-19 Testing and Clinical Outcomes Among PLWH}

It is unknown if PLWH are at increased risk for infection and severe outcomes from COVID-19. Early reports from China suggest similar rates of infection to the rest of the population [24]. However, in the United States, many PLWH come from economically disadvantaged backgrounds who are more likely to work jobs considered essential and remain at increased risk for SARS-CoV-2 exposure during social distancing $[25,26]$. Recent studies also suggest that COVID19 is more common among African American and Latinx populations, groups with higher prevalence of HIV in the US $[27,28]$. Additionally, comorbid diseases that are common among PLWH, such as cardiovascular disease may lead to worse COVID-19 outcomes [29]. Given the higher prevalence of comorbid conditions and membership in communities that suffer from structural inequities, it remains to be seen if PLWH suffer disproportionately from COVID-19.

To date, our medical center has not seen higher rates of COVID-19 among PLWH. Between March 12 and April 17, 2020, 5,537 patients were tested for COVID-19. Of these, 55 (1.0\%) were PLWH, of whom 8 tested positive for COVID-19 (14.5\%). Of HIV-negative patients tested for COVID-19, 1061/5482(19.4\%) were positive (Chi-square p-value $=0.37$ ), showing no significant difference in testing positive for COVID-19 based on HIV status. Among the 8 PLWH with COVID-19, 75\% (6/8) were hospitalized, $12.5 \%$ $(1 / 8)$ required admission to the intensive care unit, and none have died.

\section{Conclusion}

The COVID-19 pandemic has the potential to greatly disrupt HIV care continuum outcomes among PLWH. As we experience unprecedented financial stressors and necessary changes to our healthcare delivery system, we must adapt our care and services to continue to engage PLWH to avoid poor outcomes amongst our more vulnerable patients. Prioritizing patients who are not virally suppressed may serve as a successful strategy for preventing patients from falling off the HIV care continuum. Additional and timely resources are needed to provide rapid ART access and to combat food insecurity and homelessness. Healthcare services must be creatively adapted to support physical and mental health remotely to meet individual and community needs. While we are not currently seeing disproportionate COVID-19 among PLWH, we continue to monitor those outcomes. More research is needed to know the long-term implications of the pandemic for the health of people living with HIV.

\section{References}

1. Shiau S, Krause KD, Valera P, Swaminathan S, Halkitis PN. The Burden of COVID-19 in People Living with HIV: A Syndemic Perspective. AIDS Behav. Apr 182020.

2. Jiang H, Zhou Y, Tang W. Maintaining HIV care during the COVID-19 pandemic. Lancet HIV. Apr 62020.

3. Bradley H, Hall HI, Wolitski RJ, et al. Vital Signs: HIV diagnosis, care, and treatment among persons living with HIV-United States, 2011. MMWR Morb Mortal Wkly Rep. 2014;63(47):1113-7. 
4. Ulett KB, Willig JH, Lin HY, et al. The therapeutic implications of timely linkage and early retention in HIV care. AIDS patient care and STDs. 2009;23(1):41-9.

5. Mugavero MJ, Lin HY, Willig JH, et al. Missed visits and mortality among patients establishing initial outpatient HIV treatment. Clinic Infecti Dis. 2009;48(2):248-56.

6. Park WB, Choe PG, Kim SH, et al. One-year adherence to clinic visits after highly active antiretroviral therapy: a predictor of clinical progress in HIV patients. J Intern Med. 2007;261(3):268-75.

7. Centers for Disease Control and Prevention. Social Distancing, Quarantine, and Isolation. https://www.cdc.gov/coronavirus/2019ncov/prevent-getting-sick/social-distancing.html. Updated April 4, 2020. Accessed April 23, 2020.

8. Illinois Executive Order 2020-18. https://www2.illinois.gov/ Pages/Executive-Orders/ExecutiveOrder2020-18.aspx.

9. State of Indiana. Executive Order 20-22.https://www.in.gov/gov/ files/Executive\%20Order\%2020-22\%20Extension\%20of\%20Sta y\%20at $\% 20$ Home.pdf.

10. Mervosh S, Lu D, Swales V. See Which States and Cities Have Told Residents to Stay at Home2020.

11. Health Resources and Servies Administration. Ryan White \& Global HIV/AIDS Programs. Coronavirus Disease 2019 (COVID19) Frequently Asked Questions. https://hab.hrsa.gov/coronavirus/ frequently-asked-questions\#utilizing/ Updated 4/22/20. Accessed $4 / 23 / 20$.

12. Saberi P, Yuan P, John M, Sheon N, Johnson MO. A pilot study to engage and counsel HIV-positive African American youth via telehealth technology. AIDS Patient Care STDS. 2013;27(9):529-32.

13. Leon A, Caceres C, Fernandez E, et al. A new multidisciplinary home care telemedicine system to monitor stable chronic human immunodeficiency virus-infected patients: a randomized study. PLoS ONE. 2011;6(1):e14515.

14. Wootton AR, McCuistian C, Legnitto Packard DA, Gruber VA, Saberi P. Overcoming Technological Challenges: Lessons Learned from a Telehealth Counseling Study. Telemed J E Health. Dec 3 2019.

15. Ridgway JP, Almirol EA, Schmitt J, Schuble T, Schneider JA. Travel Time to Clinic but not Neighborhood Crime Rate is Associated with Retention in Care Among HIV-Positive Patients. AIDS Behav. 2018;22(9):3003-8.

16. Menton J. Record 22 million Americans file for unemployment benefits over past month as layoffs continue. USA Today. April 16,2020

17. Bing EG, Burnam MA, Longshore D, et al. Psychiatric disorders and drug use among human immunodeficiency virusinfected adults in the United States. Arch Gen Psychiatry. 2001;58(8):721-8.

18. Schumacher JE, McCullumsmith C, Mugavero MJ, et al. Routine depression screening in an HIV clinic cohort identifies patients with complex psychiatric co-morbidities who show significant response to treatment. AIDS Behav. 2013;17(8):2781-91.

19. Tegger MK, Crane HM, Tapia KA, Uldall KK, Holte SE, Kitahata MM. The effect of mental illness, substance use, and treatment for depression on the initiation of highly active antiretroviral therapy among HIV-infected individuals. AIDS Patient Care STDS. 2008;22(3):233-43.

20. Ciesla JA, Roberts JE. Meta-analysis of the relationship between HIV infection and risk for depressive disorders. Am J Psychiatry. 2001;158(5):725-30.

21. Chenneville T, Gabbidon K, Drake H, Rodriguez C. Comparison of the utility of the PHQ and CES-D for depression screening among youth with HIV in an integrated care setting. J Affect Disord. 2019;250:140-4.

22. Shacham E, Nurutdinova D, Satyanarayana V, Stamm K, Overton ET. Routine screening for depression: identifying a challenge for successful HIV care. AIDS Patient Care STDS. 2009;23(11):949-55.

23. Yao H, Chen JH, Xu YF. Patients with mental health disorders in the COVID-19 epidemic. Lancet Psychiatry. 2020;7(4):e21.

24. Guo W, Ming F, Dong Y et al. A Survey for COVID-19 among HIV/AIDS Patients in Two Districts of Wuhan, China. Preprint research paper, The Lancet, 2020.

25. Denning P, DiNenno E. Communities in Crisis: Is There a Generalized HIV Epidemic in Impoverished Urban Areas of the United States? Division of HIV/AIDS Prevention, National Center for HIV/AIDS, Viral Hepatitis, STD, and TB Prevention, Centers for Disease Control and Prevention. 2019; https://www.cdc.gov/hiv/ group/poverty.html.

26. Artiga S, Garfield R, Orgera K. Communities of Color at Higher Risk for Health and Economic Challenges due to COVID-19. Kaiser Family Foundation. . 2020; https://www.kff.org/disparitie s-policy/issue-brief/communities-of-color-at-higher-risk-for-healt h-and-economic-challenges-due-to-covid-19/.

27. Centers for Disease Control and Prevention. HIV Surveillance Report, 2018 (Preliminary); vol. 30. https://www.cdc.gov/hiv/ library/reports/hiv-surveillance.html. Published November 2019. Accessed April 23, 2020.

28. Yancy CW. COVID-19 and African Americans. JAMA. Apr 15 2020.

29. Guan WJ, Ni ZY, Hu Y, et al. Clinical Characteristics of Coronavirus Disease 2019 in China. N Engl J Med. Feb 282020.

Publisher's Note Springer Nature remains neutral with regard to jurisdictional claims in published maps and institutional affiliations. 